\title{
Exosomal MALAT1 derived from oxidized low-density lipoprotein-treated endothelial cells promotes M2 macrophage polarization
}

\author{
CHAOYANG HUANG ${ }^{*}$, JIE HAN* ${ }^{*}$ YUTAO WU, SHAN LI, QIWEN WANG, WENJUAN LIN and JIANHUA ZHU \\ Department of Cardiology, The First Affiliated Hospital of Zhejiang University School of Medicine, \\ Hangzhou, Zhejiang 310003, P.R. China
}

Received September 18, 2017; Accepted January 29, 2018

DOI: $10.3892 / \mathrm{mmr} .2018 .8982$

\begin{abstract}
Oxidized low-density lipoprotein (oxLDL)-induced injury and apoptosis of endothelial cells are important initial events in numerous cardiovascular diseases. Following activation by oxLDL, monocytes adhere to endothelial cells, migrate into the subendothelial spaces and then undergo differentiation into macrophages, which subsequently induces the formation of atherosclerotic lesions. However, the mechanisms underlying the activation of macrophage differentiation by oxLDL-treated endothelial cells remain unclear. In the present study, it was demonstrated that exosomal metastasis-associated lung adenocarcinoma transcript 1 (MALAT1) was increased in oxLDL-treated human umbilical vein endothelial cells. When co-cultured with monocytes, exosomes extracted from oxLDL-treated HUVECs were endocytosed. Furthermore, exosomes derived from oxLDL-treated endothelial cells were revealed to promote $\mathrm{M} 2$ macrophage polarization, as reverse transcription-quantitative polymerase chain reaction, western blotting and ELISA analyses demonstrated increases in the expression of M2 macrophage markers, including macrophage mannose receptor 1 (also termed CD206), arginase-1 and interleukin (IL)-10, and decreases in the expression of the M1 macrophage marker, IL-12. Furthermore, the suppression of MALAT1 expression in monocytes was demonstrated to reverse exosome-mediated M2 macrophage polarization. In conclusion, the results of the present study revealed a novel mechanism underlying the onset of atherogenesis associated
\end{abstract}

Correspondence to: Dr Chaoyang Huang, Department of Cardiology, The First Affiliated Hospital of Zhejiang University School of Medicine, 79 Qingchun Road, Hangzhou, Zhejiang 310003, P.R. China

E-mail:wzy7512@zju.edu.cn

*Contributed equally

Key words: oxidized low-density lipoprotein, exosome, long non-coding RNA, metastasis-associated lung adenocarcinoma transcript 1, M2 macrophage with endothelial cells and macrophages: Exosomal MALAT1 derived from oxLDL-treated endothelial cells promoted M2 macrophage polarization. This result may provide a novel scientific basis for the understanding of atherosclerosis progression.

\section{Introduction}

Endothelial dysfunction, which is closely associated with vascular endothelial cell injury, is involved in an array of pathophysiological processes, including coronary artery diseases, diabetes and hypertension (1-4). Oxidized low-density lipoprotein (oxLDL)-induced injury to vascular endothelial cells has been revealed to be an important initial event in atherosclerosis (5-7). It has been reported that the activation of endothelial cells by oxLDL may occur via the induction of the expression of various cell surface adhesion molecules, which mediate the rolling adhesion of leukocytes, including monocytes and T cells $(8,9)$. Following adhesion to the endothelium, leukocytes have been demonstrated to migrate into the intima where monocytes then differentiate into macrophages and induce atherosclerotic lesions (6-11). OxLDL has been reported to induce the onset of atherogenesis involving endothelial cells and macrophages (12). However, the mechanisms underlying the activation of macrophage differentiation by oxLDL-treated endothelial cells remain unclear.

Macrophages are heterogeneous and polarize into specific subsets, such as classically activated proinflammatory macrophage M1 (M1)-like and alternatively activated macrophage M2 (M2)-like macrophages (13). Different markers are employed for the identification of M1 and M2 macrophages. Markers for M1 macrophages include tumor necrosis factor- $\alpha$, inducible nitric oxide synthase, interleukin (IL)- 6 and integrin- $\alpha-X$ (also termed CD11c), while markers for M2 macrophages include macrophage mannose receptor 1 (also termed CD206), arginase-1 and resistin-like- $\alpha(14,15)$. In human atherosclerotic plaques, markers for M1 and M2 macrophages are present in the early and advanced stages of plaque development $(16,17)$. Previous studies in hypercholesterolemic mice have demonstrated that M2 macrophages reduce atherosclerosis, whereas suppression of M2 polarization enhances plaque progression (18-20). Therefore, it may be concluded that macrophage polarization is important for plaque progression in atherosclerosis. 
Exosomes are nano-sized membrane vesicles released by cells, with a diameter of 30-100 nm (21-23). Exosomes are important inter-cellular transporters, carrying bulk biomaterials to regulate the cellular functions of endothelial cells, vascular smooth muscle cells, fibroblasts and leukocytes (24-27). However, the function of exosomes secreted by oxLDL-treated endothelial cells during atherosclerosis remains to be determined.

Long non-coding RNAs (lncRNAs) are non-protein coding transcripts that are composed of $>200$ nucleotides, and studies have demonstrated that certain lncRNAs have important functions in the regulation of numerous biological processes, including cell proliferation, differentiation and death (28-30). Metastasis-associated lung adenocarcinoma transcript 1 (MALAT1) is a widely expressed single-exon lncRNA. MALAT1 was initially identified in non-small cell lung cancer and is reported to be involved in the regulation of various biological processes by functioning as a competing endogenous RNA (31-33).

Therefore, the aim of the present study was to investigate the function of exosomal IncRNA-MALAT1 in the process of oxLDL-treated endothelial cell-activated macrophage differentiation.

\section{Materials and methods}

Cell culture. THP-1 (American Type Culture Collection, Manassas, VA, USA) human monocyte cells were maintained in RPMI-1640 (Thermo Fisher Scientific, Inc., Waltham, MA, USA) and human umbilical vein endothelial cells immortalized with telomerase reverse transcriptase (HUVEC/TERT2; American Type Culture Collection) were maintained in Dulbecco's Modified Eagle's Medium/F12 (Thermo Fisher Scientific, Inc.), and each were supplemented with $10 \%$ heat-inactivated fetal bovine serum (Invitrogen; Thermo Fisher Scientific, Inc.), penicillin (100 U/ml) and streptomycin $(100 \mu \mathrm{g} / \mathrm{ml})$ in a humidified atmosphere of $5 \% \mathrm{CO}_{2}$ at $37^{\circ} \mathrm{C}$. All cells were confirmed to be free of mycoplasma contamination with the MycoFluor ${ }^{\mathrm{TM}}$ Mycoplasma Detection kit (Thermo Fisher Scientific, Inc.).

Exosome isolation and co-culture with THP-1 cells. In order to isolate exosomes, HUVECs $\left(2 \times 10^{7}\right)$ were treated with $50 \mu \mathrm{g} / \mathrm{ml}$ oxLDL (Shanghai Luwen Biotechnology Co., Ltd., Shanghai, China) for $48 \mathrm{~h}$ at $37^{\circ} \mathrm{C}$ and the supernatant was collected. The supernatant was centrifuged twice $(1,000 \mathrm{x} \mathrm{g}$ for $10 \mathrm{~min}$ and $3,000 \mathrm{x} \mathrm{g}$ for $30 \mathrm{~min}$ at $4 \mathrm{C}$ ) to remove the cells and fragments, added to the Total Exosome Isolation kit (Thermo Fisher Scientific, Inc.) overnight and further centrifuged at $10,000 \mathrm{x}$ g for $1 \mathrm{~h}$ at $4^{\circ} \mathrm{C}$. Exosomes were resuspended in PBS and stored at $-80^{\circ} \mathrm{C}$. The concentration of exosomes was determined using a bicinchoninic acid assay. Exosomes $(50 \mathrm{ng} / \mathrm{ml})$ were subsequently added to THP-1 cells $\left(1 \times 10^{5}\right)$ in culture medium for $24 \mathrm{~h}$ at $37^{\circ} \mathrm{C}$. Exosomes were stained with PHK67 (cat. no. MINI67; Sigma-Aldrich; Merck KGaA, Darmstadt, Germany) to observe endocytosis, according to the manufacturer's protocol.

Transmission electron microscopic observation of exosomes. The exosome suspension was added to an equal volume of
$4 \%$ paraformaldehyde at $4^{\circ} \mathrm{C}$ for $30 \mathrm{~min}$ and applied to a Formvar/Carbon film-coated transmission electron microscope grid (Alliance Biosystems, Inc., Osaka, Japan). Subsequently, the sample was fixed by incubation with $1 \%$ glutaraldehyde for $5 \mathrm{~min}$ at $25^{\circ} \mathrm{C}$, washed with PBS and incubated with $1 \%$ uranyl acetate for $5 \mathrm{~min}$ at $25^{\circ} \mathrm{C}$. The sample was subsequently observed under a transmission electron microscope (Hitachi H7650; Hitachi, Ltd., Tokyo, Japan).

Knockdown of MALAT1 in THP-1 cells. The knockdown sequence for MALAT1 was as follows: 5'-ACGGAAGTAATT CAAGATCAA-3'. Lentiviral vectors for human MALAT1 knockdown were constructed by Shanghai Hanyin Industrial Co., Ltd. (Shanghai, China). The recombinant lentivirus and the empty vector negative control (NC) lentivirus (Shanghai Hanyin Industrial Co., Ltd.) were prepared and titrated to $10^{9}$ transfection U/ml. To isolate stably transfected cell lines, THP-1 cells were seeded in 6-well plates at a density of $2 \times 10^{5}$ cells/well and infected with the same titer of virus (Shanghai Hanyin Industrial Co., Ltd., Shanghai, China) with $8 \mu \mathrm{g} / \mathrm{ml}$ polybrene (Shanghai Hanyin Industrial Co., Ltd.) on the following day at $37^{\circ} \mathrm{C}$. At $\sim 72 \mathrm{~h}$ post-viral infection, the RPMI-1640 culture medium was replaced with selection medium containing $4 \mu \mathrm{g} / \mathrm{ml}$ puromycin and the cells were cultured for $\geq 14$ days at $37^{\circ} \mathrm{C}$. The puromycin-resistant cells were amplified in medium containing $2 \mu \mathrm{g} / \mathrm{ml}$ puromycin for $7-9$ days $37^{\circ} \mathrm{C}$ and then transferred to medium without puromycin. The knockdown efficiency was confirmed via reverse transcription-quantitative polymerase chain reaction (RT-qPCR) $24 \mathrm{~h}$ after co-culture, as described below.

Western blot analysis. Protein concentration was determined using the bicinchoninic acid protein assay method. Membranes were blocked in $5 \%$ non-fat milk at $25^{\circ} \mathrm{C}$ for $30 \mathrm{~min}$. To analyze the expression of the markers CD9, CD63 and CD206 in exosomes (control or oxLDL-treated) or THP-1 cells that were co-cultured with exosomes (control or oxLDL-treated), western blot assays were performed using the following primary antibodies: Mouse anti-human CD63 (cat. no. ab59479; 1:1,000; Abcam, Cambridge, UK), rabbit anti-human CD9 (cat. no. ab92726; 1:1,000; Abcam), rabbit anti-human CD206 (cat. no. 18704-1-AP; 1:1,000; ProteinTech Group, Inc., Chicago, IL, USA) and mouse anti-actin (cat. no. MAB1501; 1:10,000; EMD Millipore, Billerica, MA, USA). Briefly, exosomes or cells were lysed with radioimmunoprecipitation assay buffer [50 mM Tris- $\mathrm{HCl}$ (pH 7.5), $150 \mathrm{mM} \mathrm{NaCl,} 1 \%$ Triton X-100 and $0.5 \%$ Na-deoxycholate] containing protease inhibitors (Complete $^{\mathrm{TM}}$, Mini protease inhibitor cocktail; Sigma-Aldrich; Merck KGaA). A total of 20-30 $\mu \mathrm{g}$ lysate were separated on 8-12\% SDS-PAGE gels and transferred to polyvinylidene fluoride membranes. The membranes were subsequently incubated with the aforementioned primary antibodies overnight at $4{ }^{\circ} \mathrm{C}$. The primary antibody incubation was followed by incubation with the following secondary antibodies at room temperature for $1 \mathrm{~h}$ : Horseradish peroxidase (HRP)-conjugated anti-rabbit (cat. no. 7074; 1:10,000; CST Biological Reagents Co., Ltd., Shanghai, China) and HRP-conjugated anti-mouse (cat. no. 7076; 1:10,000; CST Biological Reagents Co., Ltd.). The bound antibodies were detected using an enhanced chemiluminescence kit (cat. no. PI32209; Pierce; Thermo Fisher 
A

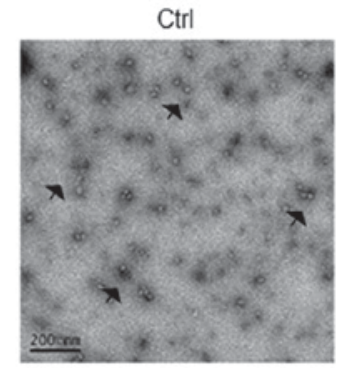

oxLDL-treated

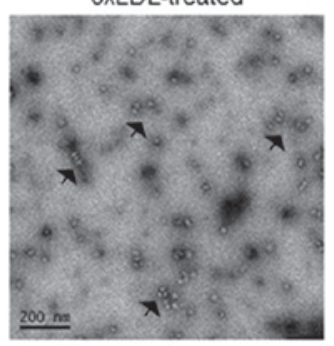

B

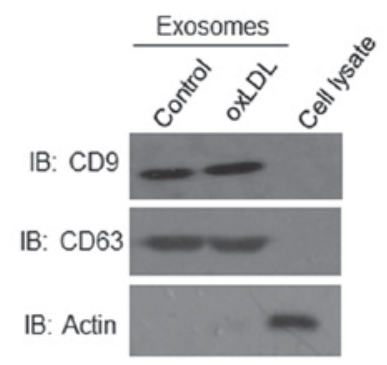

C

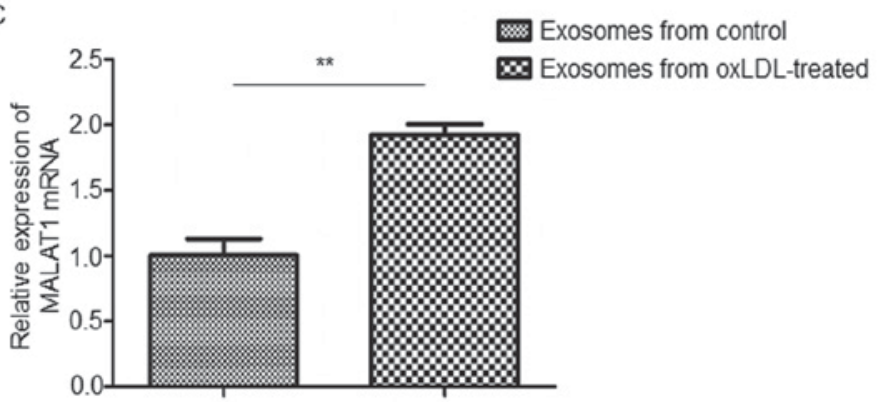

Figure 1. Exosomal MALAT1 is increased in oxLDL-treated HUVEC cells. (A) Representative electron micrographs of exosomes isolated from oxLDLtreated or untreated HUVECs, revealing the typical morphology and size $(30-120 \mathrm{~nm}$ ). Scale bar, $200 \mathrm{~nm}$. Arrows indicate the exosomes. (B) Western blot analysis confirmed the expression of CD9 and CD63, and the absence of actin, in exosomes derived from oxLDL-treated and untreated HUVECs. (C) Reverse transcription-quantitative polymerase chain reaction analysis demonstrated the expression of MALAT1 in exosomes derived from oxLDL-treated or untreated HUVECs. ${ }^{* *} \mathrm{P}<0.01$, as indicated. MALAT1, metastasis-associated lung adenocarcinoma transcript 1; oxLDL, oxidized low-density lipoprotein; HUVECs, human umbilical vein endothelial cells; Ctrl, control.

Scientific, Inc.). Quantity One analysis software (version 4.6.9; Bio-Rad Laboratories, Inc., Hercules, CA, USA) was used to quantify the relative band intensities from western blot images.

$R T-q P C R$. Total RNA was extracted from exosomes (control or oxLDL-treated) or THP-1 cells that were co-cultured with exosomes (control or oxLDL-treated) using TRIzol (Invitrogen; Thermo Fisher Scientific, Inc.). An equal quantity of total RNA was used for first-strand cDNA synthesis using the reverse transcription system (cat. no. A3500; Promega Corporation, Madison, WI, USA) according to the manufacturer's protocol. The synthesized first-strand cDNA (2 $\mu \mathrm{l}$ ) was used for each qPCR assay. A SYBR-Green PCR Master Mix (Applied Biosystems; Thermo Fisher Scientific, Inc.) was used for qPCR experiments. GAPDH was used as an internal reference control. The primers sequences were as follows: MALAT1 forward, 5'-TTGTAGACTGGAGAAGAT AGG-3' and reverse, 5'-ACTGAAGAGCATTGGAGAT-3'; CD206 forward, 5'-GGGTTGCTATCACTCTCTATGC-3' and reverse, 5'-TTTCTTGTCTGTTGCCGTAGTT-3'; Arg-1 forward, 5'-TGGACAGACTAGGAATTGGCA-3' and reverse, 5'-CCAGTCCGTCAACATCAAAACT-3'; GAPDH forward, 5'-CATGGCCTTCCGTGTTCCTA-3' and reverse 5'-GCG GCACGTCAGATCCA-3'. qPCR cycling conditions consisted of initial denaturation for $3 \mathrm{~min}$ at $95^{\circ} \mathrm{C}$, followed by 45 cycles of $95^{\circ} \mathrm{C}$ for $10 \mathrm{sec}$ and $58^{\circ} \mathrm{C}$ for $45 \mathrm{sec}$ in a LightCycler ${ }^{\circledR} 480$ instrument (Roche Diagnostics, Basel, Switzerland). Melt curve analysis was performed at the end of each run between $58-95^{\circ} \mathrm{C}$. Data were analyzed using Microsoft Excel 2013 (Microsoft Corporation, Redmond, WA, USA). The $2^{-\Delta \Delta \mathrm{Cq}}$ relative quantification method was used to analyze quantitative RT-qPCR data using the housekeeping gene GAPDH for normalization (34).
ELISA. ELISAs were performed on THP-1 cells that had been co-cultured with exosomes from control or oxLDL-treated HUVECs to determine the effect on IL-10 and IL-12 levels. IL-10 and IL-12 ELISA kits were purchased from Abcam (cat. nos. ab185986 and ab46035, respectively), and the analysis was performed according to the manufacturer's protocol.

Statistical analysis. Statistical differences between two groups were determined using the Student's t-test. Experiments were repeated three times. Data are presented as the mean \pm standard error of the mean. SPSS software was used to perform statistical analysis (version 17.0; SPSS, Inc., Chicago, IL, USA). $\mathrm{P}<0.05$ was considered to indicate a statistically significant difference.

\section{Results}

Exosomal MALAT1 is increased in oxLDL-treated HUVECs. To investigate the functions of oxLDL-treated HUVECderived exosomes in atherosclerosis, exosomes were isolated from oxLDL-treated or untreated HUVECs. The morphology of isolated exosomes was observed under transmission electron microscopy (Fig. 1A). As revealed in Fig. 1A, the diameters of the exosomes ranged from 30-120 nm. Furthermore, the results of western blot analysis demonstrated that, compared with cell lysates, exosomes were enriched with CD9 and CD63 exosomal markers (Fig. 1B), thus confirming the effective isolation of exosomes.

Following this, levels of MALAT1 mRNA expression were investigated in isolated exosomes via RT-qPCR, and the results revealed that exosomes secreted by oxLDL-treated HUVECs exhibited significantly enhanced levels of MALAT1 mRNA compared with those excreted by untreated controls (Fig. 1C). 
A

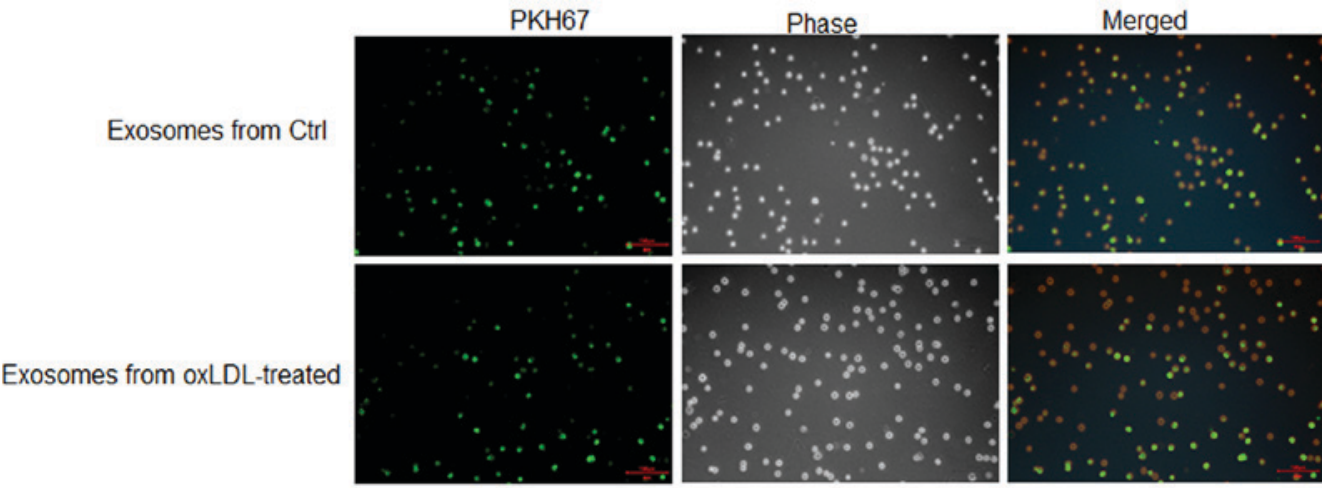

B

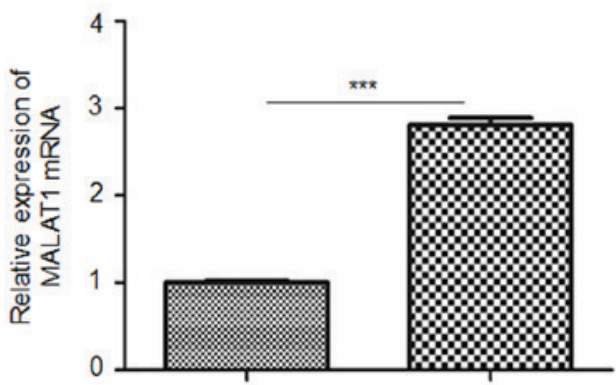

Exosomes from control

$\mathbf{\infty}$ Exosomes from oxLDL-treated

Figure 2. Monocytes endocytose exosomes derived from HUVECs. (A) Fluorescent microscopy observation results of exosomes isolated from oxLDL-treated or untreated HUVECs co-cultured with THP-1 cells. Exosomes were stained with PHK67 (green). Magnification, x200. (B) Reverse transcription-quantitative polymerase chain reaction analysis revealed the expression of MALAT1 in THP-1 cells co-cultured with exosomes derived from oxLDL-treated or untreated HUVECs. ${ }^{* * * *} \mathrm{P}<0.001$, as indicated. HUVECs, human umbilical vein endothelial cells; oxLDL, oxidized low-density lipoprotein; MALAT1, metastasisassociated lung adenocarcinoma transcript 1; Ctrl, control.

Monocytes endocytose exosomes isolated from ox $L D L$ treated HUVECs. The present study investigated whether exosomes released from oxLDL-treated HUVECs could be taken up by monocytes. Exosomes labeled with PHK67 (green) were co-cultured with THP-1 cells. Following $24 \mathrm{~h}$ of co-culture, THP-1 cells were fixed and observed by fluorescence microscopy. As revealed in Fig. 2A, exosomes were observed in co-cultured THP-1 cells, thus indicating that monocytes successfully endocytosed exosomes derived from untreated and oxLDL-treated HUVECs. Furthermore, the levels of MALAT1 mRNA in co-cultured THP-1 cells were investigated via RT-qPCR. The RT-qPCR results demonstrated that THP-1 cells co-cultured with exosomes secreted by oxLDL-treated HUVECs exhibited significantly enhanced levels of MALAT1 mRNA compared with those co-cultured with exosomes excreted by untreated control HUVECs (Fig. 2B).

Exosomes derived from oxLDL-treated HUVECs promote M2 macrophage polarization. In order to investigate the effects of oxLDL-treated HUVEC-derived exosomes on macrophage polarization, THP-1 cells were co-cultured with exosomes isolated from oxLDL-treated or untreated HUVECs and the expression of M1 and M2 macrophage markers were investigated by RT-qPCR, western blotting and ELISA analyses. The results demonstrated that oxLDL-treated HUVEC-derived exosomes enhanced the expression of the M2 macrophage markers (CD206, Arg-1 and IL-10; Fig. 3A-E) compared with oxLDL-untreated HUVEC-derived exosomes, whereas the expression of the M1 macrophage marker (IL-12; Fig. 3F) was reduced compared with oxLDL-untreated HUVEC-derived exosomes. These results indicate that exosomes derived from oxLDL-treated endothelial cells promoted M2 macrophage polarization.

Suppression of MALAT1 expression reverses exosomemediated M2 macrophage polarization. To further verify the effect of exosomal MALAT1 on macrophage polarization, MALAT1 expression was knocked down in THP-1 cells and successful knockdown was confirmed by RT-qPCR (Fig. 4A). oxLDL-treated HUVEC-derived exosomes were co-cultured with THP-1 cells with or without MALAT1 knockdown. The results of subsequent RT-qPCR, western blotting and ELISA analyses revealed that knockdown of MALAT1 in THP-1 cells co-cultured with oxLDL-treated HUVEC-derived exosomes led to the reduced expression of M2 macrophage markers (CD206, Arg-1 and IL-10; Fig. 4B-F) and increased levels of IL-12, an M1 macrophage marker (Fig. 4G), compared with those co-cultured with oxLDL-treated HUVEC-derived exosomes without knockdown of MALAT1. These results indicate that the suppression of MALAT1 expression reversed M2 macrophage polarization mediated by oxLDL-treated HUVEC-derived exosomes.

\section{Discussion}

Recent studies have demonstrated that exosomes mediate intercellular communication via the transfer of RNAs and proteins, which have important roles in numerous physiological and pathological processes, including cardiovascular 

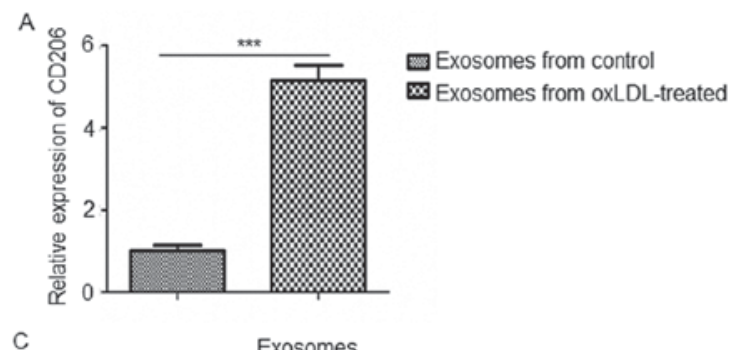

C

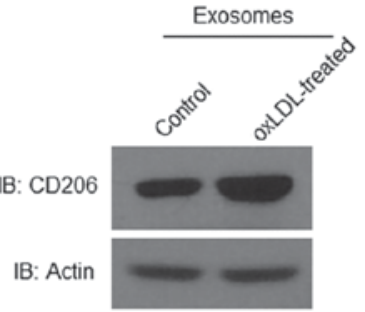

$\mathrm{E}$

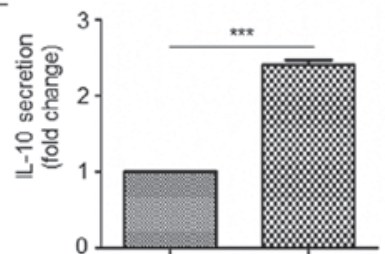

$\square$ Exosomes from control

Exosomes from oxLDL-treated
B

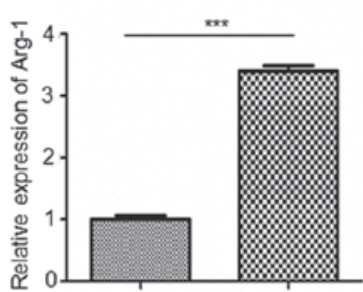

D

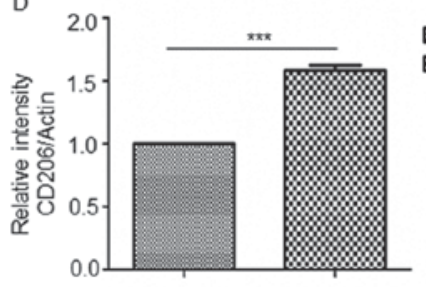

Exosomes from control

$\times$ Exosomes from oxLDL-treated
Exosomes from control

$\propto$ Exosomes from oxLDL-treated

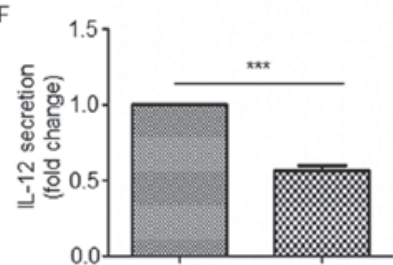

Figure 3. Exosomes derived from oxLDL-treated HUVECs promote M2 macrophage polarization. Reverse transcription-quantitative polymerase chain reaction analyses revealed an increase in the mRNA expression of (A) CD206 and (B) Arg-1 in THP-1 cells co-cultured with exosomes derived from oxLDLtreated HUVECs. (C) Representative western blot bands for CD206 protein expression in THP-1 cells co-cultured with exosomes derived from oxLDL-treated or untreated HUVECs. (D) Western blot analysis revealed an increase in the protein expression of CD206 in THP-1 cells co-cultured with exosomes derived from oxLDL-treated HUVECs. ELISA results demonstrated (E) an increase in IL-10 secretion and (F) a decrease in IL-12 secretion in THP-1 cells co-cultured with exosomes derived from oxLDL-untreated HUVECs. ${ }^{* * * *} \mathrm{P}<0.001$, as indicated. oxLDL, oxidized low-density lipoprotein; HUVECs, human umbilical vein endothelial cells; Arg-1, arginase-1; IL, interleukin.

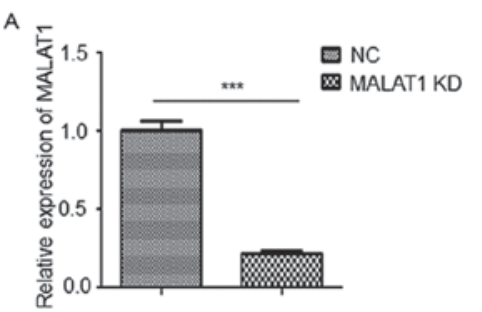

D
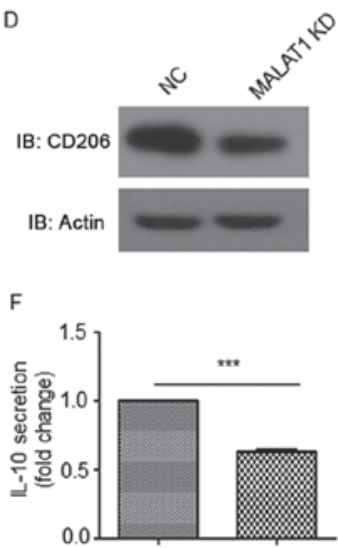

B

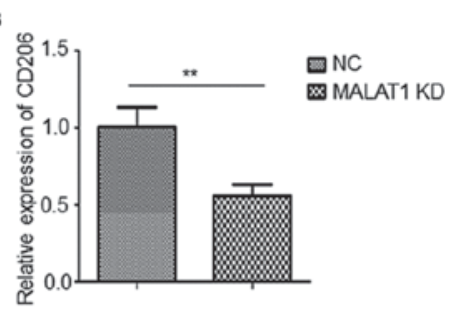

E

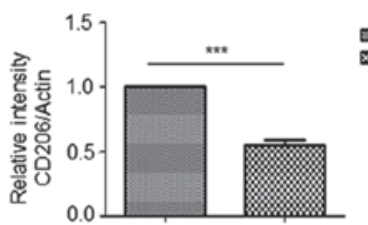

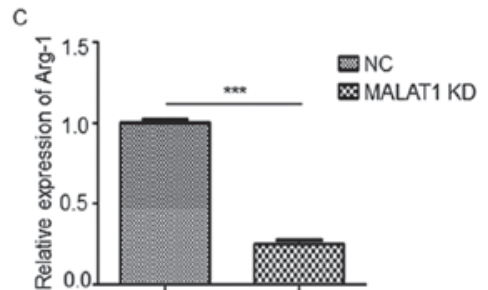

$\square \mathrm{NC}$

MALAT1 KD
Exosomes from control

Exosomes from oxLDL-treated 
disease (21-27). Chen et al (35) reported that exosomal lncRNA growth arrest-specific 5 regulated the apoptosis of macrophages and vascular endothelial cells in atherosclerosis. In the present study, it was revealed that the expression of exosomal MALAT1 was enhanced in oxLDL-treated HUVECs. Furthermore, the results of the present study revealed that, following co-culture with monocytes, these exosomes were endocytosed and promoted M2 macrophage polarization via enhanced expression of the M2 macrophage markers CD206, Arg-1 and IL-10, and reduced expression of the M1 macrophage marker, IL-12. Additionally, it was demonstrated that the suppression of MALAT1 expression in monocytes reversed M2 macrophage polarization mediated by oxLDL-treated HUVEC-derived exosomes.

OxLDL contributes to atherosclerotic progression via numerous mechanisms, including the induction of endothelial cell dysfunction and macrophage foam cell formation (12). In addition, oxLDL-induced injury in the retinal pigment epithelium was reported to enhance the exosomal and apoptotic bleb release of CD46 and CD59, which are membrane complement regulatory factors, thus indicating that oxLDL stimulation may affect exosomal release (36). Reciprocal interactions between endothelial cells and macrophages have been revealed in angiogenic vascular niches via secreted microvesicles, such as exosomes (37). In the present study, it was demonstrated that oxLDL-stimulated HUVEC-derived exosomes mediated the polarization of macrophages. In addition, in recent studies, exosomes from numerous cell types, including stem cells and cancer cells, have been demonstrated to be involved in macrophage polarization (38-40). However, the underlying molecular mechanisms of this process remain unknown and require further investigation.

It has previously been established that exosomes contain mRNAs, non-coding RNAs and proteins (23-25). It was previously reported that exosomes derived from hypoxic epithelial ovarian cancer deliver microRNA-940 for the induction of M2 macrophage polarization (39). Additionally, exosomal MALAT1 has been reported to have important roles in the regulation of numerous pathological processes, including those involved in cancer and endocrinology (41-43). Serum MALAT-1 exosomal expression was upregulated and demonstrated to promote cell proliferation and migration in non-small cell lung cancer (42). Huangfu et al (44) demonstrated that oxLDL induced MALAT1 transcription through the nuclear factor- $\kappa \mathrm{B}$ pathway. Furthermore, Tang et al (45) revealed that MALAT1 protects the endothelium from ox-LDL-induced endothelial dysfunction, in part through competition with miR22-3p for endogenous CXCR2 and AKT mRNA. The results of the present study indicate a novel mechanism for the onset of atherogenesis involving endothelial cells and macrophages. M2 macrophages have atheroprotective function by preventing foam cell formation (17). Thus, the results of the present study demonstrate that exosomal MALAT1 derived from oxLDLtreated endothelial cells may promote M2 macrophage polarization, and this may provide a novel scientific basis for the understanding of atherosclerosis progression.

\section{Acknowledgements}

Not applicable.

\section{Funding}

This research was supported by the Natural Sciences Foundation of Zhejiang Province (grant nos. LY17H020003 and LY17H020006) and Zhejiang Medical Science and Technology Project (grant no. 2015KYA070).

\section{Availability of data and materials}

All data generated or analyzed during this study are included in this published article.

\section{Authors' contributions}

$\mathrm{CH}$ and $\mathrm{JH}$ designed the study, acquired, analyzed and interpreted the data, and wrote the manuscript. YW, SL, QW and WL collected and analyzed the data. JZ was involved in designing the experiments, interpreting data, and drafting and revising the manuscript.

\section{Ethics approval and consent to participate}

Not applicable.

\section{Consent for publication}

Not applicable.

\section{Competing interests}

The authors declare that they have no competing interests.

\section{References}

1. Gkaliagkousi E, Gavriilaki E, Triantafyllou A and Douma S: Clinical significance of endothelial dysfunction in essential hypertension. Curr Hypertens Rep 17: 85, 2015.

2. Prieto D, Contreras C and Sánchez A: Endothelial dysfunction, obesity and insulin resistance. Curr Vasc Pharmacol 12: 412-426, 2014.

3. de Jager SCA, Meeuwsen JAL, van Pijpen FM, Zoet GA, Barendrecht AD, Franx A, Pasterkamp G, van Rijn BB, Goumans MJ and den Ruijter HM: Preeclampsia and coronary plaque erosion: Manifestations of endothelial dysfunction resulting in cardiovascular events in women. Eur J Pharmacol 816: 129-137, 2017.

4. Yang X, Li Y, Li Y, Ren X, Zhang X, Hu D, Gao Y, Xing Y and Shang H: Oxidative stress-mediated atherosclerosis: Mechanisms and therapies. Front Physiol 8: 600, 2017.

5. Chen M, Ren L, Meng Y, Shi L, Chen L, Yu B, Wu Q and Qi G: The protease inhibitor E64d improves ox-LDL-induced endothelial dysfunction in human aortic endothelial cells. Can J Physiol Pharmacol 96: 120-127, 2018.

6. Li J, Liang X, Wang Y, Xu Z and Li G: Investigation of highly expressed PCSK9 in atherosclerotic plaques and ox-LDL-induced endothelial cell apoptosis. Mol Med Rep 16: 1817-1825, 2017.

7. Li Z, Huang H, Huang L, Du L, Sun Y and Duan Y: Prevention of oxidized low density lipoprotein-induced endothelial cell injury by DA-PLGA-PEG-cRGD nanoparticles combined with ultrasound. Int J Mol Sci 18: pii: E815, 2017.

8. Amberger A, Maczek C, Jürgens G, Michaelis D, Schett G, Trieb K, Eberl T, Jindal S, Xu Q and Wick G: Co-expression of ICAM-1, VCAM-1, ELAM-1 and Hsp60 in human arterial and venous endothelial cells in response to cytokines and oxidized low-density lipoproteins. Cell Stress Chaperones 2: 94-103, 1997.

9. Weber C, Erl W, Weber KS and Weber PC: Effects of oxidized low density lipoprotein, lipid mediators and statins on vascular cell interactions. Clin Chem Lab Med 37: 243-251, 1999. 
10. Di Pietro N, Formoso G and Pandolfi A: Physiology and pathophysiology of oxLDL uptake by vascular wall cells in atherosclerosis. Vascul Pharmacol 84: 1-7, 2016.

11. Westhorpe CL, Dufour EM, Maisa A, Jaworowski A, Crowe SM and Muller WA: Endothelial cell activation promotes foam cell formation by monocytes following transendothelial migration in an in vitro model. Exp Mol Pathol 93: 220-226, 2012.

12. Pirillo A, Norata GD and Catapano AL: LOX-1, OxLDL, and atherosclerosis. Mediators Inflamm 2013: 152786, 2013.

13. Wang $\mathrm{N}$, Liang $\mathrm{H}$ and Zen $\mathrm{K}$ : Molecular mechanisms that influence the macrophage $\mathrm{m} 1-\mathrm{m} 2$ polarization balance. Front Immunol 5: 614, 2014.

14. Bories GFP and Leitinger N: Macrophage metabolism in atherosclerosis. FEBS Lett 591: 3042-3060, 2017.

15. Liberale L, Dallegri F, Montecucco F and Carbone F: Pathophysiological relevance of macrophage subsets in atherogenesis Thromb Haemost 117: 7-18, 2017.

16. Chistiakov DA, Bobryshev YV, Nikiforov NG, Elizova NV Sobenin IA and Orekhov AN: Macrophage phenotypic plasticity in atherosclerosis: The associated features and the peculiarities of the expression of inflammatory genes. Int J Cardiol 184 436-445, 2015.

17. Colin S, Chinetti-Gbaguidi G and Staels B: Macrophage phenotypes in atherosclerosis. Immunol Rev 262: 153-166, 2014.

18. Fadini GP, Simoni F, Cappellari R, Vitturi N, Galasso S, Vigili de Kreutzenberg S, Previato L and Avogaro A: Pro-inflammatory monocyte-macrophage polarization imbalance in human hypercholesterolemia and atherosclerosis. Atherosclerosis 237 : 805-808, 2014

19. Xu R, Li C, Wu Y, Shen L, Ma J, Qian J and Ge J: Role of KCa3.1 channels in macrophage polarization and its relevance in atherosclerotic plaque instability. Arterioscler Thromb Vasc Biol 37: 226-236, 2017.

20. Lin Y, Chen Z and Kato S: Receptor-selective IL-4 mutein modulates inflammatory vascular cell phenotypes and attenuates atherogenesis in apolipoprotein E-knockout mice. Exp Mol Pathol 99: 116-127, 2015.

21. Martínez MC and Andriantsitohaina R: Extracellular vesicles in metabolic syndrome. Circ Res 120: 1674-1686, 2017.

22. Todorova D, Simoncini S, Lacroix R, Sabatier F and Dignat-George F: Extracellular vesicles in angiogenesis. Circ Res 120: 1658-1673, 2017.

23. Nomura S: Extracellular vesicles and blood diseases. Int J Hematol 105: 392-405, 2017.

24. Goetzl EJ, Schwartz JB, Mustapic M, Lobach IV, Daneman R, Abner EL and Jicha GA: Altered cargo proteins of human plasma endothelial cell-derived exosomes in atherosclerotic cerebrovascular disease. FASEB J 31: 3689-3694, 2017.

25. Lu X: The role of exosomes and exosome-derived microRNAs in atherosclerosis. Curr Pharm Des: Apr 13, 2017 (Epub ahead of print).

26. Niu C, Wang X, Zhao M, Cai T, Liu P, Li J, Willard B, Zu L, Zhou E, Li Y, et al: Macrophage foam cell-derived extracellular vesicles promote vascular smooth muscle cell migration and adhesion. J Am Heart Assoc 5: pii: e00409, 2016.

27. Cai J, Wu G, Jose PA and Zeng C: Functional transferred DNA within extracellular vesicles. Exp Cell Res 349: 179-183, 2016.

28. Liu Y, Zheng L, Wang Q and $\mathrm{Hu}$ YW: Emerging roles and mechanisms of long noncoding RNAs in atherosclerosis. Int $\mathrm{J}$ Cardiol 228: 570-582, 2017.

29. $\mathrm{Li} \mathrm{H}, \mathrm{Zhu} \mathrm{H}$ and $\mathrm{Ge} \mathrm{J}$ : Long noncoding RNA: Recent updates in atherosclerosis. Int J Biol Sci 12: 898-910, 2016.
30. Zhou T, Ding JW, Wang XA and Zheng XX: Long noncoding RNAs and atherosclerosis. Atherosclerosis 248: 51-61, 2016.

31. Zhang X, Hamblin MH and Yin KJ: The long noncoding RNA malat1: Its physiological and pathophysiological functions. RNA Biol 14: 1705-1714, 2017.

32. Uchida S and Dimmeler S: Long noncoding RNAs in cardiovascular diseases. Circ Res 116: 737-750, 2015.

33. Yoshimoto R, Mayeda A, Yoshida M and Nakagawa S: MALAT1 long non-coding RNA in cancer. Biochim Biophys Acta 1859: 192-199, 2016.

34. Livak KJ and Schmittgen TD: Analysis of relative gene expression data using real-time quantitative PCR and the 2(-Delta Delta C(T)) method. Methods 25: 402-408, 2001.

35. Chen L, Yang W, Guo Y, Chen W, Zheng P, Zeng J and Tong W: Exosomal lncRNA GAS5 regulates the apoptosis of macrophages and vascular endothelial cells in atherosclerosis. PLoS One 12: e0185406, 2017.

36. Ebrahimi KB, Fijalkowski N, Cano M and Handa JT: Oxidized low-density-lipoprotein-induced injury in retinal pigment epithelium alters expression of the membrane complement regulatory factors CD46 and CD59 through exosomal and apoptotic bleb release. Adv Exp Med Biol 801: 259-265, 2014.

37. Baer C, Squadrito ML, Iruela-Arispe ML and De Palma M Reciprocal interactions between endothelial cells and macrophages in angiogenic vascular niches. Exp Cell Res 319: 1626-1634, 2013

38. Zhao H, Shang Q, Pan Z, Bai Y, Li Z, Zhang H, Zhang Q, Guo C, Zhang $L$ and Wang Q: Exosomes from adipose-derived stem cells attenuate adipose inflammation and obesity through polarizing M2 macrophages and beiging in white adipose tissues. Diabetes 67: 235-247, 2018

39. Chen X, Ying X, Wang X, Wu X, Zhu Q and Wang X: Exosomes derived from hypoxic epithelial ovarian cancer deliver microRNA-940 to induce macrophage M2 polarization. Oncol Rep 38: 522-528, 2017.

40. Song Y, Dou H, Li X, Zhao X, Li Y, Liu D, Ji J, Liu F, Ding L, Ni Y and Hou Y: Exosomal miR-146a contributes to the enhanced therapeutic efficacy of interleukin-1 $\beta$-primed mesenchymal stem cells against sepsis. Stem Cells 35: 1208-1221, 2017.

41. Gezer U, Özgür E, Cetinkaya M, Isin M and Dalay N: Long noncoding RNAs with low expression levels in cells are enriched in secreted exosomes. Cell Biol Int 38: 1076-1079, 2014.

42. Zhang R, Xia Y, Wang Z, Zheng J, Chen Y, Li X, Wang Y and Ming H: Serum long non coding RNA MALAT-1 protected by exosomes is up-regulated and promotes cell proliferation and migration in non-small cell lung cancer. Biochem Biophys Res Commun 490: 406-414, 2017.

43. El Bassit G, Patel RS, Carter G, Shibu V, Patel AA, Song S, Murr M, Cooper DR, Bickford PC and Patel NA: MALAT1 in human adipose stem cells modulates survival and alternative splicing of PKCסII in HT22 cells. Endocrinology 158: 183-195, 2017.

44. Huangfu N, Xu Z, Zheng W, Wang Y, Cheng J and Chen X: LncRNA MALAT1 regulates oxLDL-induced CD36 expression via activating $\beta$-catenin. Biochem Biophys Res Commun 495: 2111-2117, 2018

45. Tang Y, Jin X, Xiang Y, Chen Y, Shen CX, Zhang YC and Li YG: The lncRNA MALAT1 protects the endothelium against ox-LDL-induced dysfunction via upregulating the expression of the miR-22-3p target genes CXCR2 and AKT. FEBS Lett 589: 3189-3196, 2015. 